\title{
Asymmetric stem-cell division ensures sustained keratinocyte hyperproliferation in psoriatic skin lesions
}

\author{
HAI-YAN JIA, YING SHI, LONG-FEI LUO, GUAN JIANG, QIONG ZHOU, SHI-ZHENG XU and TIE-CHI LEI \\ Department of Dermatology, Wuhan University, Renmin Hospital, Wuhan, Hubei 430060, P.R. China
}

Received July 5, 2015; Accepted December 14, 2015

DOI: $10.3892 / \mathrm{ijmm} .2015 .2445$

\begin{abstract}
Excessive expansion of the transit-amplifying (TA) cell compartment is a distinct morphological characteristic of psoriatic epidermal hyperplasia. In order to examine the activation of basal stem cells and how they replenish such an enlarged compartment of TA cells in psoriatic epidermis, we utilized a BrdU labeling method to monitor mitotic stem cells in a mouse model of psoriasiform dermatitis, which was induced by imiquimod. Our results showed that perpendicular and parallel cell division characteristics of dividing stem cells existed in the inflamed epidermis. When we analyzed template-DNA strand segregation in trypsin-dissociated human psoriatic keratinocytes using BrdU pulse-chase labeling, we found that the percentage of asymmetric segregation of BrdU was significantly increased in the cell pairs of psoriatic epidermal cells compared with normal epidermal cells. Furthermore, we also examined the effects of both interleukin (IL)-17A and IL-22 cytokines on the differentiation status of cultured human keratinocytes. The results indicated that both cytokines had synergistic effects on passage-one epidermal cell sheets derived from skin explants and also on cultured keratinocytes, were involved in the maintenance of the undifferentiated stem cell phenotype, and these results suggest an efficient mechanism for preventing the premature loss of basal stem-cell pools in the pro-inflammatory cytokine-enriched milieu of the psoriatic epidermis. Our findings suggest that inhibition of hyperactive stem cells represents a potential therapeutic target to combat recalcitrant epidermal hyperplasia in psoriasis.
\end{abstract}

\section{Introduction}

Psoriasis is a chronic, relapsing, papulosquamous dermatitis characterized by abnormal hyperproliferation of the epidermis. It affects approximately $2 \%$ of the population and all racial groups $(1,2)$. The prominent cutaneous manifestations of psoriasis present as raised, well-demarcated, erythematous

Correspondence to: Dr Tie-Chi Lei, Department of Dermatology, Wuhan University, Renmin Hospital, 238 Jiefang Road, Wuchang, Wuhan, Hubei 430060, P.R. China

E-mail: tchlei@whu.edu.cn

Key words: keratinocyte, mitosis, interleukin, stem cell, psoriasis plaques with adherent silvery scales. The scales result from the hyperproliferative epidermis, the premature maturation of keratinocytes, as well as the incomplete cornification and retention of nuclei in the stratum corneum (parakeratosis). The mitotic rate of basal keratinocytes in psoriatic lesions is significantly increased compared with normal skin (1). Consequently, psoriasis has long been considered only a disease of the keratinocytes which involves basal cell hyperproliferation $(3,4)$. However, substantial advances have been made in terms of elucidating the molecular mechanisms of psoriasis, and previous studies have demonstrated that the disease is a disorder resulting from the dysregulated interplay between keratinocytes and infiltrating immune cells (5-7).

In previous research, an array of pro-inflammatory cytokines have been detected in psoriatic skin lesions, which have been demonstrated to act as major drivers of acanthosis in psoriasis. Of these cytokines, interleukin (IL)-17A and IL-22 appear to be the most active cytokines in the immunopathogenesis of psoriasis (8) and also in cases of imiquimod (IMQ)-induced psoriasiform dermatitis in mice $(3,6)$. The functional receptors (IL-17RA and IL-22R) for those cytokines are constitutively expressed on the surface of keratinocytes $(8,9)$. Increased production of IL-17A and IL-22, through infiltration of Th17 cells, has been reported in psoriatic skin lesions. These cytokines act on keratinocytes by binding to their cognate receptors, activating the basal keratinocytes from a quiescent state into a hyperproliferative state, retarding the terminal differentiation of keratinocytes, and driving the infiltration of inflammatory cells such as neutrophils into the epidermis (9). Intradermal injection of recombinant IL-17A and IL-22 in a mouse model of allogeneic skin-humanized psoriasis resulted in epidermal hyperplasia and mixed inflammatory cell infiltrates, features which closely resembled the majority of those of human psoriasis (10).

The epidermis is an avascular and multilayered epithelium composed of a single layer of proliferative basal cells and several suprabasal (or spinous) layers of differentiated keratinocytes (11). It has been noted that there are two distinct subpopulations of proliferative keratinocytes in the basal layer: stem cells, which have an unlimited capacity for self-renewal (but are thought to proliferate infrequently and to be generally quiescent) and also transit-amplifying (TA) cells (the descendants of stem cells, which are destined to withdraw from the cell cycle and terminally differentiate after a few rounds of division) (12-15). It is tempting to hypothesize that activated 
stem cells give rise to the extreme expansion of TA cells in the psoriatic epidermis, but it remains uncertain whether hyperproliferative psoriatic keratinocytes causes the exhaustion and/or reduction of the stem cell pool. Our aims for the present study were as follows: i) to investigate the asymmetric cell division of trypsin-dissociated human psoriatic keratinocytes and the proportion of mitotic basal cells in the mouse model of dermatitis induced by the immune activator IMQ, using pulse-chase labeling with bromodeoxyuridine (BrdU) to understand the transition of stem cells to TA cells as well as post-mitotic (PM) cells; and ii) to examine the effects of the Th17-derived cytokines IL-17A and IL-22 on the expression of differentiation-related markers both in keratinocytes and in passage-one epidermal cell sheets derived from skin explants (16), which are of value in determining the role of the maintenance of the stem-cell pool in the pro-inflammatory cytokine-enriched milieu of psoriatic epidermis.

\section{Materials and methods}

Chemicals, cytokines and antibodies. All chemicals were obtained from Sigma-Aldrich (St. Louis, MO, USA) unless otherwise stated. Recombinant human IL-17A and IL-22 were purchased from PeproTech (Rocky Hill, NJ, USA). The following monoclonal antibodies (mAbs) were used: anti-BrdU antibody (BU-33; B2531) was obtained from Sigma-Aldrich, anti-BrdU antibody conjugated with FITC (ab74545) was purchased from Abcam (Cambridge, MA, USA), antibodies against differentiation-specific markers, namely K15 (ab52816; Abcam), K10 (SC31770; Santa Cruz Biotechnology, Inc., Santa Cruz, CA, USA), integrin $\beta 1 /$ FITC (ab46920), filaggrin (ab24584), and Ki67 (ab16667) were all purchased from Abcam. Secondary antibodies conjugated with Alexa Fluor 488 (green; A11001) or 555 (red; A21432) were purchased from Molecular Probes (Eugene, OR, USA). Mowiol 4-88 anti-fade mounting solution was obtained from Polyscience, Inc. (Warrington, PA, USA).

Patients. Eleven patients suffering from psoriasis vulgaris and ten healthy controls were enrolled in this study. The clinical and demographic characteristics of the subjects included in this study were summarized. Certain patients had a family history of the disease (data not shown). At the time that skin biopsies were taken, medications had been discontinued for several months. Samples were obtained under local anesthesia from psoriasis skin plaques using a 6-mm biopsy punch. All patients were recruited from the Department of Dermatology (Renmin Hospital of Wuhan University, Wuhan, China), and normal skin samples were collected from patients who underwent breast reconstruction in the Department of Plastic Surgery (Renmin Hospital of Wuhan University). This study was conducted in accordance with the Declaration of Helsinki Principles, and was approved by the Ethics Committee of Renmin Hospital of Wuhan University.

Mouse model and BrdU injections. BALB/c male mice (8-11 weeks old) were purchased from the animal facility of Hubei Provincial Center for Disease Control and Prevention (CDC; Wuhan, China), raised in pathogen-free conditions and provided with food and water ad libitum. All experiments were approved by the Animal Ethics Committee according to Chinese legislation on animal experiments. Mice were treated daily with commercially available IMQ cream (5\%) (Aldara; 3M Pharmaceuticals, Minneapolis, MN, USA), which induced psoriasiform dermatitis, on shaved dorsal skin, or treated with a control cream on shaved dorsal skin for 6 consecutive days as previously reported (6). BrdU incorporation was performed to monitor the mitotic activity of basal cells in the inflamed skin. A single dose of BrdU at $50 \mu \mathrm{g} / \mathrm{gram}$ body weight was administered to mice by intraperitoneal injection $2 \mathrm{~h}$ prior to mice being sacrificed by carbon dioxide inhalation. Dorsal skin was biopsied and fixed in buffered formalin and embedded in paraffin. Five-micrometer sections were cut on a microtome and stained with mouse-anti-BrdU (Sigma-Aldrich) and a commercially available streptavidin-peroxidase (SP) staining kit (Maixin-Bio, Fuzhou, China) in order to visualize BrdU immunostaining in the paraffin-embedded sections.

Cell cultures and treatments. As previously described (17), the skin tissue was trimmed to remove subcutaneous adipose tissue and cut into small strips. These skin strips were soaked in Dulbecco's modified Eagle's medium (DMEM) (Gibco, Carlsbad, CA, USA) containing $0.25 \%$ dispase (Sigma-Aldrich) and digested overnight at $4^{\circ} \mathrm{C}$. The following day, the epidermis was peeled off and further dissociated after simple digestion in $0.25 \%$ trypsin. Single cell cultures were obtained by passing the cell suspension through a nylon cell strainer with $70-\mu \mathrm{m}$ pore size (Becton-Dickinson, Franklin Lakes, NJ, USA) and then washing with PBS. Primary cells were maintained in EpiLife keratinocyte medium with $1 \%$ (v/v) human keratinocyte growth supplement (both from Invitrogen, Camarillo, CA, USA) and $60 \mu \mathrm{M}$ calcium. Cells at an early passage $(\leq 5)$ were used for all experiments. To analyze the expression pattern of differentiation-related proteins, the calcium concentration was increased to $1.3 \mathrm{mM}$ in cultures. The primary cells were also stimulated with recombinant IL-17A (10 ng/ml) and IL-22 $(10 \mathrm{ng} / \mathrm{ml})$ for $24 \mathrm{~h}$ before protein extraction $(17,18)$.

Pulse-chase BrdU labeling to assess asymmetric cell division. For labeling of cells in culture, the cells were plated at a low density $\left(\sim 10-20\right.$ cells $\left./ \mathrm{mm}^{2}\right)$ in 6 -well tissue culture plates which contained a collagen (Sigma-Aldrich)-coated coverslip in each well, as previously described $(19,20)$. After cells had attached, $10 \mu \mathrm{M}$ BrdU (B9285; Sigma-Aldrich) was added for $30 \mathrm{~h}$ of labeling. Subsequently, cell cultures were washed with PBS twice and replaced with fresh medium containing $5 \mu \mathrm{M}$ cytochalasin D (C8273; Sigma-Aldrich) to arrest cytokinesis. After a chase period of $30 \mathrm{~h}$, cells on the coverslips were fixed with $4 \%$ paraformaldehyde solution for subsequent $\mathrm{BrdU}$ immunostaining.

Preparation of passage-one epidermal cell sheets. Passage one epidermal cell sheets were prepared following a previously reported protocol (16). Briefly, skin tissue samples were trimmed to remove subcutaneous tissue and were further cut into small strips using ophthalmologic scissors. Skin strips were placed on glass coverslips at the bottom of 6-well plates with the epidermal side facing upwards. After allowing the skin pieces to firmly attach to the coverslips, DMEM containing $20 \%$ fetal bovine serum (FBS) and antibiotics $(0.625 \mu \mathrm{g} / \mathrm{ml}$ 
amphotericin B, $100 \mathrm{IU} / \mathrm{ml}$ penicillin and $100 \mu \mathrm{g} / \mathrm{ml}$ streptomycin) were carefully added to the plates, ensuring no tissue pieces floated upwards. After 3-5 days of cultivation, passage one epidermal cells had grown out from the edge of each skin explant to form a tongue-like epidermal sheet, as visualized in Fig. 6A. The epidermal sheet cultures were treated with recombinant IL-17A $(10 \mathrm{ng} / \mathrm{ml})$ and IL-22 $(10 \mathrm{ng} / \mathrm{ml})$ for $24 \mathrm{~h}$ to further analyze the expression pattern of differentiation-related proteins. Finally, the skin pieces were removed gently using an ophthalmologic forceps and fixed with $4 \%$ paraformaldehyde solution for subsequent immunofluorescence staining.

Immunohistochemical and immunofluorescence assays. In the present study, formalin-fixed paraffin sections $(5 \mu \mathrm{M})$ were dewaxed by melting for $30-60 \mathrm{~min}$ at $60^{\circ} \mathrm{C}$, and they were cleared in xylene 3 times for 5 min each and then rehydrated in water bath solutions containing decreasing percentages of ethanol. The sections were then treated to inhibit endogenous peroxidases, blocked and incubated overnight at $4^{\circ} \mathrm{C}$ with specific primary antibodies against proliferation and/or differentiation-related protein markers: anti-BrdU (dilution 1:100), anti-K15 (1:100), anti-K10 (1:100), anti-integrin $\beta 1$ (1:50), anti-filaggrin (1:100) and anti-Ki67 (1:100). After incubation with the primary antibodies, sections were rinsed with Tris-buffered solution (TBS) and were then incubated with a biotinylated secondary antibody (dilution 1:2,000; Maixin-Bio, Fuzhou, China) for $1 \mathrm{~h}$. The immune signals were detected using a commercially available SP staining kit (Maixin-Bio). Sections were counterstained with hematoxylin and eosin and mounted. The number of positively immunostained cells in the basal layers (aside from K10 and Ki67 in the suprabasal layer) was counted from at least 6 randomly selected fields under a magnification of $x 400$ and expressed as a percentage of the total cells counted. For BrdU immunofluorescence staining, cell cultures were fixed in 70\% ethanol, washed with PBS, and incubated with $4 \mathrm{M} \mathrm{HCl}$ for $10 \mathrm{~min}$ at room temperature, and then washed 3 times with PBS containing 1\% FBS and $0.01 \%$ sodium azide. They were then incubated with anti-BrdU/FITC antibody for $45 \mathrm{~min}$ at $37^{\circ} \mathrm{C}$. The sections were counterstained with 4',6-diamidino-2-phenylindole (DAPI) (Invitrogen) or propidium iodide (Sigma-Aldrich) to identify the nuclei. The routine protocol was also carried out for the detection of intracellular and extracellular epitopes, as detailed in the figure legends. Double immunofluorescence was performed with anti-BrdU/FITC (dilution 1:100) and anti-K15 (1:50). Slides were mounted with Mowiol 4-88 anti-fade mounting solution. Immunostaining was subsequently observed using an Olympus IX71 fluorescence microscope.

Western blotting. Cells were washed in PBS and lysed in extraction buffer containing $1 \%$ Nonidet P-40, $0.01 \%$ SDS and a protease inhibitor cocktail (Roche, Indianapolis, IN, USA). Protein content was determined with a BCA assay kit (Pierce, Rockford, IL, USA). Equal amounts of each protein extract (30 $\mu \mathrm{g} / \mathrm{lane}$ ) were then resolved by using $6 \%$ sodium dodecyl sulfate-polyacrylamide gel electrophoresis (SDS-PAGE). Following blotting onto Immobilon-P membranes (Millipore, Bedford, MA, USA) and blocking with 5\% non-fat milk in saline buffer, the membranes were incubated with anti-K15, anti-K10, anti-integrin, anti-filaggrin, and anti-Ki67, each at a

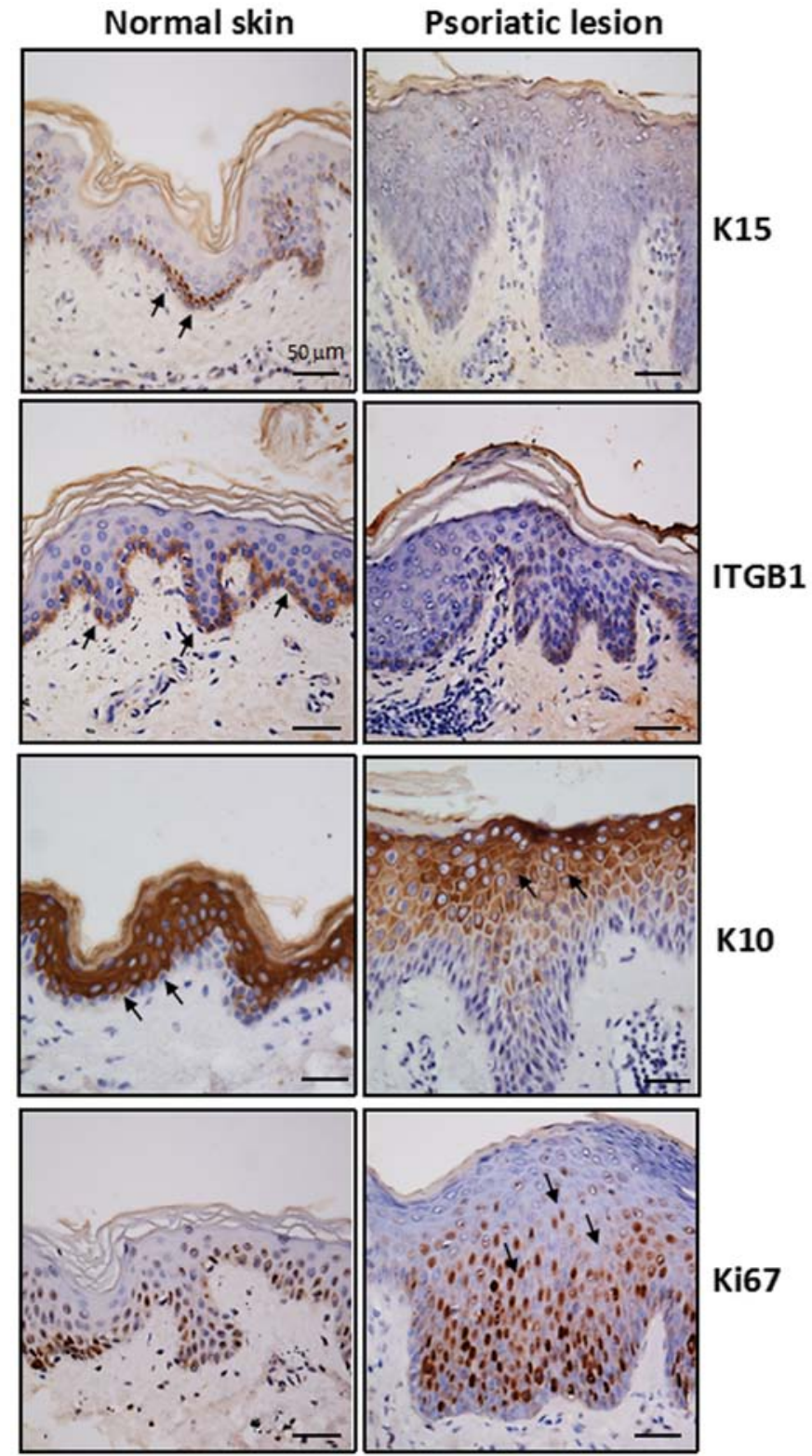

Figure 1. Enlarged compartments of transit-amplifying (TA) cells in psoriatic plaques. The expression profiles of markers for stem cells (K15), TA cells (integrin $\beta 1$ ), and post-mitotic (PM) cells (K10) as well as the cellular proliferative marker $(\mathrm{Ki} 67)$ were detected in normal skin $(\mathrm{n}=5)$ and in psoriatic plaque tissues $(n=5)$ using routine immunohistochemical analysis. Depicted are representative images of the enlarged compartments of TA cells (suprabasal spinous cells) in a psoriatic plaque (right panels), corresponding to normal skin tissue (left panels). Arrows indicate the germinative zone, which contains proliferating TA cells in psoriatic plaque tissues. Scale bars, $50 \mu \mathrm{m}$.

1:2,000 dilution, or with an anti-GAPDH antibody (Santa Cruz Biotechnology, Inc.) at a dilution of 1:1,000, for $1 \mathrm{~h}$ at room temperature. The membranes were then washed and incubated with horseradish-peroxidase-conjugated anti-rabbit $\operatorname{IgG}$ (Pierce) at a dilution of 1:2,000 for $1 \mathrm{~h}$ at room temperature. Membranes were then washed again, and specific bands were visualized using enhanced chemiluminescence (ECL) reaction (Amersham, Piscataway, NJ, USA).

Statistical analysis. The Student's t-test was used to compare the average band intensities resulting from western blotting, the average epidermal thickness and the average cell-pair 
A control

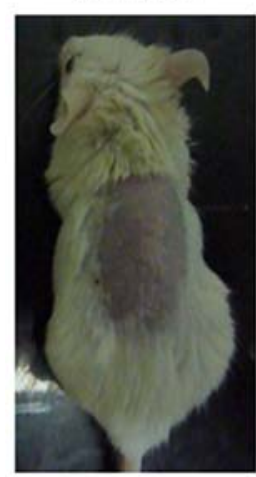

IMQ

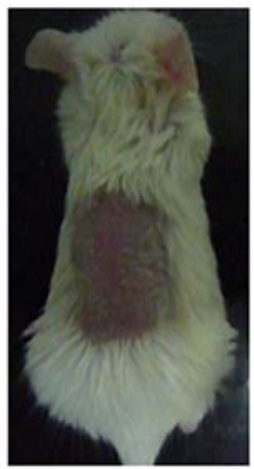

B

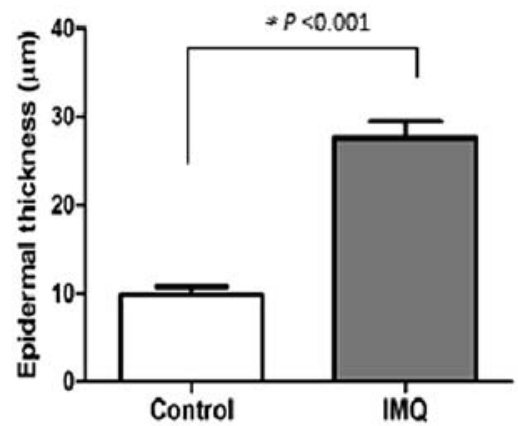

C

$\mathrm{H} \& \mathrm{E}$
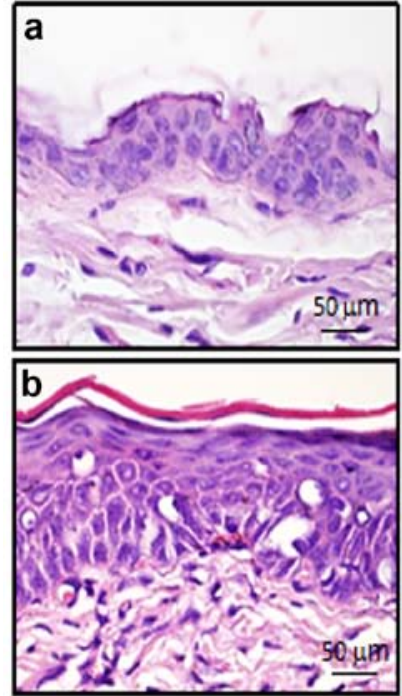

Ki67
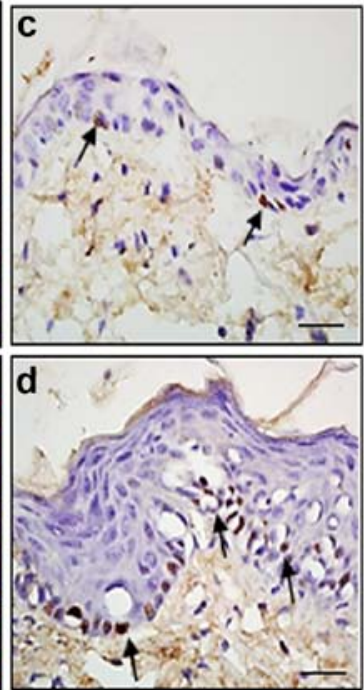

BrdU

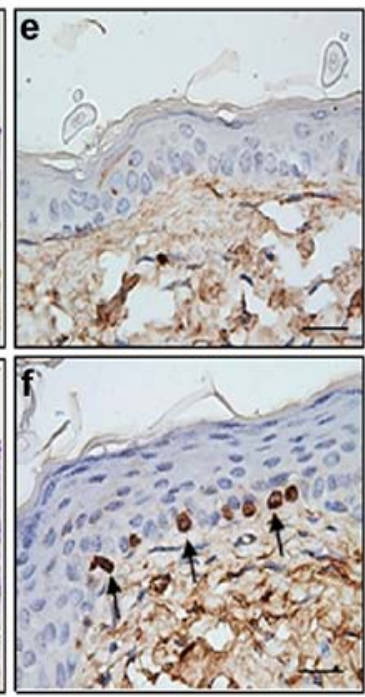

Figure 2. Mouse psoriasiform dermatitis induced by imiquimod (IMQ). BALB/c mice (3 mice/group) were treated daily with IMQ cream or control cream on the shaved dorsal skin for 6 days, as detailed in Materials and methods. (A) Phenotypical presentation of mouse dorsal skin after treatment. (B) Epidermal thickness of the dorsal skin was quantified using ImageJ densitometry software. Positive cells were counted as described in Materials and methods. Data are represented as the means \pm SEM from 3 independent experiments. ${ }^{*} \mathrm{P}<0.001$, treatment groups compared with controls. (C) Representative images of (a and b) hematoxylin and eosin (H\&E) staining and (c and d) Ki67 immunostaining of the dorsal skin treated with IMQ (b and d) and control (a and c) are shown. Arrows indicate Ki67-positive cells. To track mitotic cells in vivo, 2-3 mice/group were injected with BrdU $2 \mathrm{~h}$ before sacrifice. BrdU incorporation in keratinocytes in the dorsal skin treated by (e) control and (f) IMQ and was detected by immunohistochemistry. Arrows indicate BrdU-labeled cells. Scale bar, $50 \mu$ m.

number of asymmetric cell divisions. Epidermal thickness of the dorsal skin was quantifed using ImageJ densitometry software (NIH, Bethesda, MD, USA) (21). Groups were compared using ANOVA. In the present study, a P-value $<0.05$ (5\%) was considered to indicate a statistically significant difference and was denoted with asterisks. SPSS software for Windows version 11.0 was used to analyze the results.

\section{Results}

Aberrant activation of basal stem cells causes excessive expansion of the TA cell compartment in the psoriatic epidermis. To fully characterize the histological alterations of psoriatic TA cell expansion, we first analyzed the expression of phenotypic markers relevant to proliferation and differentiation in psoriatic and normal skin samples. We used immunohistochemical analysis and a panel of specific antibodies against putative stem cells (K15 and integrin $\beta 1$ ), TA cells (integrin $\beta 1$ ) and PM cells (K10) as well as a cellular proliferative marker (Ki67) (Fig. 1). The immunostaining results listed in Table I indicate the percentage of positive cells in the psoriatic lesions and the normal epidermis. K15 expression was significantly decreased in the psoriatic epidermis $(11.7 \pm 3.2 \%)$ compared with normal skin $(43.3 \pm 12.6 \%, \mathrm{P}<0.05)$. Immunostaining for Ki67 revealed a markedly increased proportion of stained cells in the suprabasal layer, but the emergence of K10-positive keratinocytes was postponed until they reached the granular layer. By contrast to the immunostaining pattern of K10 in the psoriatic epidermis, intensive staining for K10 was clear in the entire suprabasal layer in the normal epidermis, aside from the single basal cell layer that did not express K10. These findings suggest that excessive expansion of the TA cell population 
A
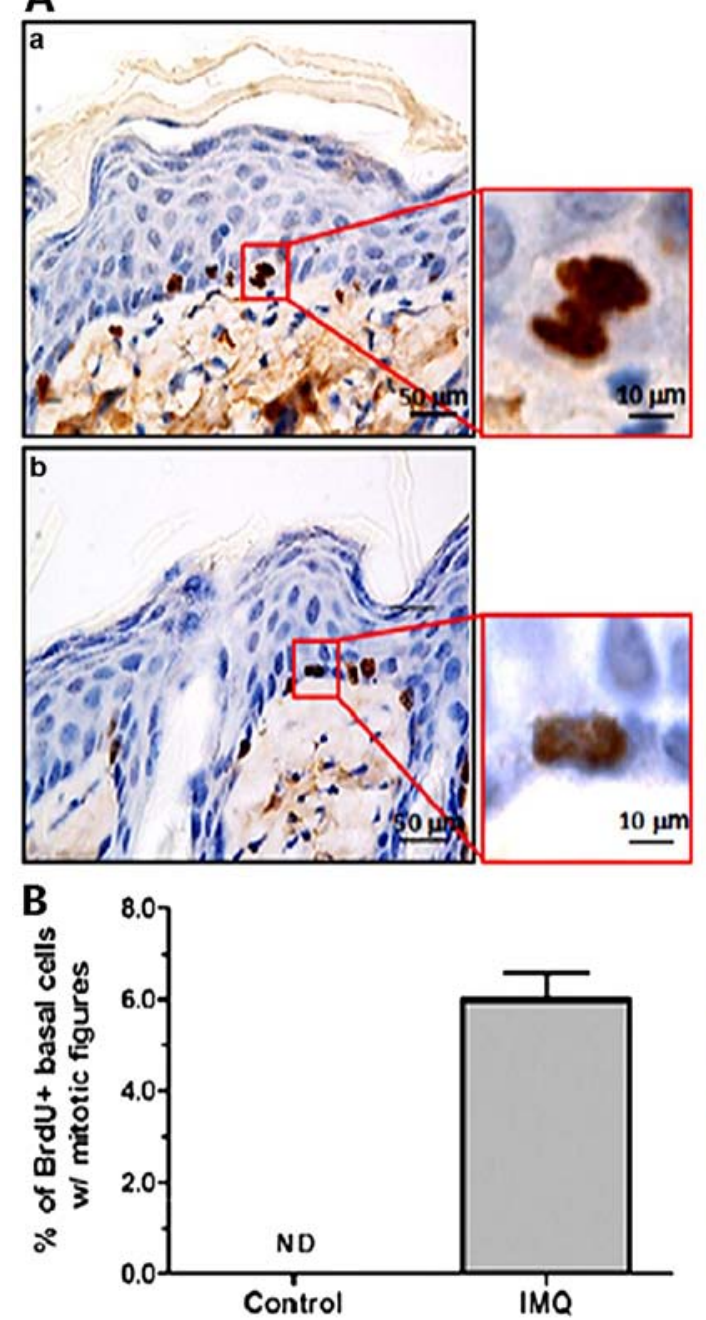

C

Epidermis of normal skin

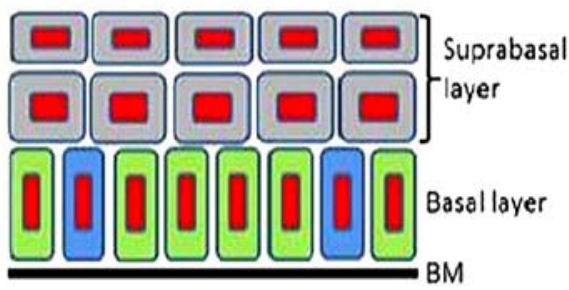

Epidermis of Psoriatic plaque

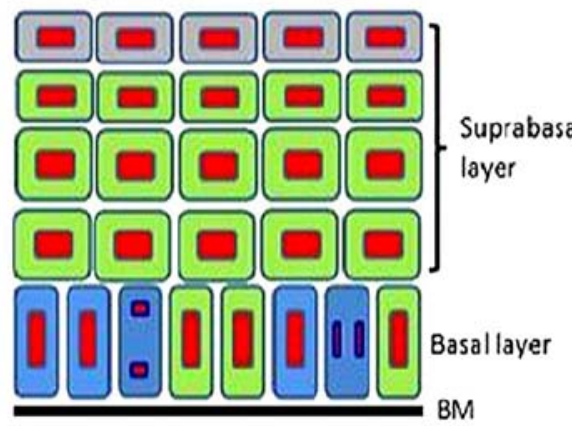

Stem cell (K15+/ITGB1++/Ki67.)

Transit amplifying cell (K15-/ITGB1+/Ki67+)

Post-mitotic cell (K10+/ITGB1-/Ki67-)

a

Perpendicularcell division

00 Parallel cell division

Figure 3. Measurement of mitotic basal cells in imiquimod (IMQ)-induced psoriasis-like dermatitis in mice by bromodeoxyuridine (BrdU)-labeling. BALB/c mice were treated daily with IMQ cream or control cream on shaved dorsal skin for 6 days. To track mitotic cells in vivo, 2-3 mice/group were injected with BrdU $2 \mathrm{~h}$ before euthanasia. (A) BrdU incorporation in keratinocytes in the dorsal skin was detected by immunohistochemistry. Representative images of the perpendicular or parallel cell division of mitotic cells are shown in the left panels, and high-magnification images of the boxed-in areas are shown on the right. (B) Bar graph showing the percentage of BrdU-labeled cells with mitotic figures from 3 independent experiments with at least 100 cells counted per experiment. (C) Depictions of perpendicular and parallel cell division in mice with psoriasis and control mice. ND, none detected.

Table I. Immunostaining analysis of proliferation and differentiation of keratinocytes in the psoriatic and normal epidermis (means \pm SEM)

\begin{tabular}{lcccc}
\hline Groups & K15 (\%) & ITGB1 (\%) & K10 (\%) & Ki67 (\%) \\
\hline Normal skin & $43.3 \pm 12.6$ & $94.6 \pm 6.6$ & $93.3 \pm 4.1$ & $22.3 \pm 8.0$ \\
Psoriatic lesion & $11.7 \pm 3.2$ & $33.6 \pm 9.7$ & $60.6 \pm 15.5$ & $53.6 \pm 8.7$ \\
P-value & 0.025 & 0.0008 & 0.036 & 0.029
\end{tabular}

The positive cells were counted in the basal layer for K15/ITGB1 and in the suprabasal layer for K10/Ki67. ITGB1, integrin $\beta 1$.

existed in the psoriatic epidermis, which may have exhausted the stem cell pool to some extent since K15-positive cells were barely detectable in the psoriatic basal layer. Considering that in vivo lineage tracing to directly monitor changes of the stem cell pool is not feasible in humans (12), we thus measured the number of mitotic basal cells using BrdU labeling in the mouse model of IMQ-induced dermatitis (Fig. 2), which is an animal model simulating some clinical features of human psoriasis (6). Representative stained images of BrdU-labeled basal cells are shown in Fig. 3. A proportion $(6 \%)$ of BrdU-labeled mitotic basal cells was easily detected in the inflamed skin of the mice, but those cells were negligible in the control mice. Interestingly, two types of asymmetric cell division, perpendicular and parallel (17), were clearly discerned in BrdU-labeled basal cells (Fig. 3). These data indicate that the quiescent basal cells become activated to undergo cell division, which may serve as a prelude to epidermal hyperplasia in this model of psoriasis.

Both symmetric and asymmetric cell division is increased in psoriatic epidermal cells. Based on the above observation that K15 immunostaining was significantly reduced in psoriatic basal cells, we further examined whether there was a reduction of the stem cell pool in cases of psoriasis. It should be noted, however, that one may speculate that decreased K15 expression contributes to the active states of stem cells $(22,23)$. First, the 

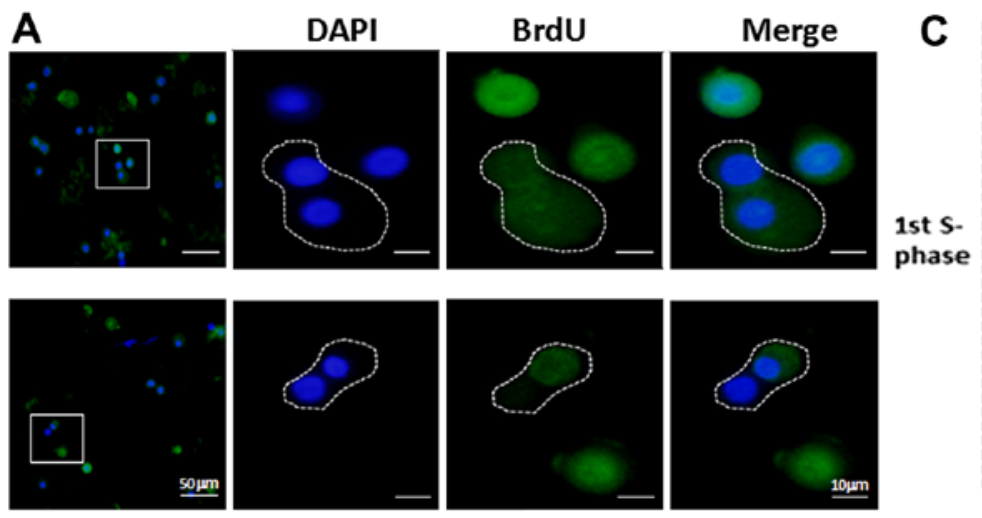

B
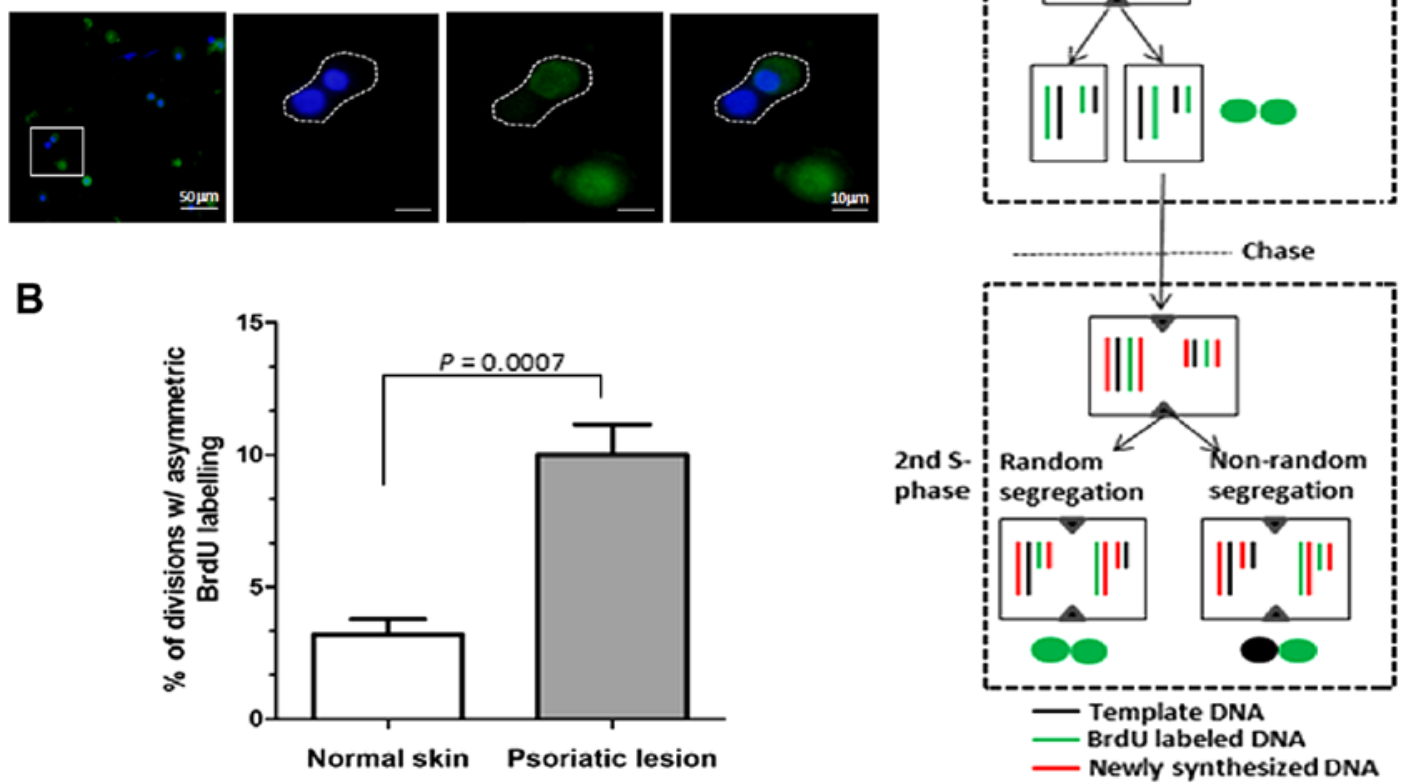

Figure 4. In vitro analyses for template-DNA strand co-segregation in trypsin-dissociated psoriatic keratinocytes using BrdU pulse-chase labeling. Primary keratinocytes (passage 2) were selectively cultured from psoriatic plaques and from normal skin tissues, and were then plated singly on collagen-coated coverslips in 6-well tissue culture plates. After the cells had attached, $10 \mu \mathrm{M} \mathrm{BrdU}$ was added for a $30 \mathrm{~h}$ pulse-labeling (the doubling time for keratinocytes). The cells were washed and re-fed with fresh medium containing $5 \mu \mathrm{M}$ cytochalasin $\mathrm{D}$ for an additional $30 \mathrm{~h}$ to arrest cytokinesis. After the chase period, the cells were fixed for BrdU immunostaining. The slides were counterstained with DAPI to identify nuclei. Immunostaining was observed using an Olympus IX71 fluorescence microscope. (A) Cell pairs were visualized for BrdU (green) and DAPI (blue). Depicted is a representative image of an immunostained pair of cells: the top row shows a cell pair with symmetric inheritance of BrdU-labeled DNA, and the bottom row shows an asymmetric pair. The dotted lines in the top and bottom rows delineate one cell pair each. The areas boxed in white in the low-magnification images (the far left panels) indicate the locations of the cell pairs in the slide. The percentage of cells presenting asymmetric inheritance of template DNA strands is shown in (B). Data represent the means \pm SD from 3 independent experiments. "P<0.05, compared to normal keratinocytes. (C) Schematic view of the immortal DNA strand hypothesis. Template DNA strands (black) segregate to one daughter cell (right) compared with random chromatid segregation (left) in the second S-phase.

BrdU pulse-chase labeling protocol was employed to identify template-DNA strand segregation during mitosis in primary cultures of psoriatic epidermal cells. In general, daughter cells inheriting the older template DNA are considered stem cells according to the immortal strand hypothesis $(19,20)$. The data demonstrated that an increased proportion $(\sim 10 \%)$ of asymmetric segregation of BrdU was noted in the cell pairs of psoriatic epidermal cells, whereas only $3 \%$ was noted in normal epidermal cells $(\mathrm{P}<0.001)$ (Fig. 4). This suggests that hyperactive asymmetric stem cell division exists in cultured psoriatic keratinocytes. Secondly, the correlation between asymmetric cell division and the transition from stem cells to TA cells was also investigated by examination of BrdU pulse-chased anaphase cells using dual immunostaining for both BrdU segregation and K15 expression. The results showed that primary keratinocytes underwent asymmetric division in which the template DNA (unlabeled) always segregated to the daughter cell that retained K15, as shown in Fig. 5A. The percentage ( 13\%) of cells expressing K15 that were asymmetrically labeled with $\mathrm{BrdU}\left(\mathrm{BrdU} / \mathrm{K}^{+} 5^{+} ; \mathrm{BrdU}^{+} /\right.$ $\mathrm{K} 15$; putative asymmetric stem cell division in the second round) was increased in psoriatic keratinocytes compared with normal cells $(\mathrm{P}<0.05)$, as shown in Fig. 5B (bottom row). The percentage $(32 \%)$ of cells expressing K15 that were symmetrically labeled with $\mathrm{BrdU}\left(\mathrm{BrdU}^{+} / \mathrm{K} 5^{+} ; \mathrm{BrdU}^{+++}\right.$; putative stem cell self-renewal in the first round) was also increased in psoriatic keratinocytes compared with normal cells $(\mathrm{P}<0.01)$, as shown in Fig. 5B (top row). It seems safe to conclude that the increased proportion of both symmetric and asymmetric cell division may provide a mechanism by which the psoriatic epidermis maintains persistent hyperplastic plaques.

\section{IL-17A and IL-22 inhibit keratinocyte differentiation to} maintain the stem cell phenotype. To explore the effects of IL-17A and IL-22 on basal cells expressing stem cell markers characteristic for K15 and the integrin $\beta 1$, we first established a short-term culture system that allowed passage-one epidermal cells migrating from skin explants to form an epidermal cell sheet, as shown in Fig. 6A. We then treated these tongue-like epithelial sheets with $10 \mathrm{ng} / \mathrm{ml}$ recombinant IL-17A plus $10 \mathrm{ng} / \mathrm{ml} \mathrm{IL-22}$ for $24 \mathrm{~h}$ in vitro. The pattern of K15 and integrin $\beta 1$ expression was then examined in those epithelial sheets by immunofluorescence staining. The results showed that cells expressing K15 and/or integrin $\beta 1$ were restricted to the leading- 

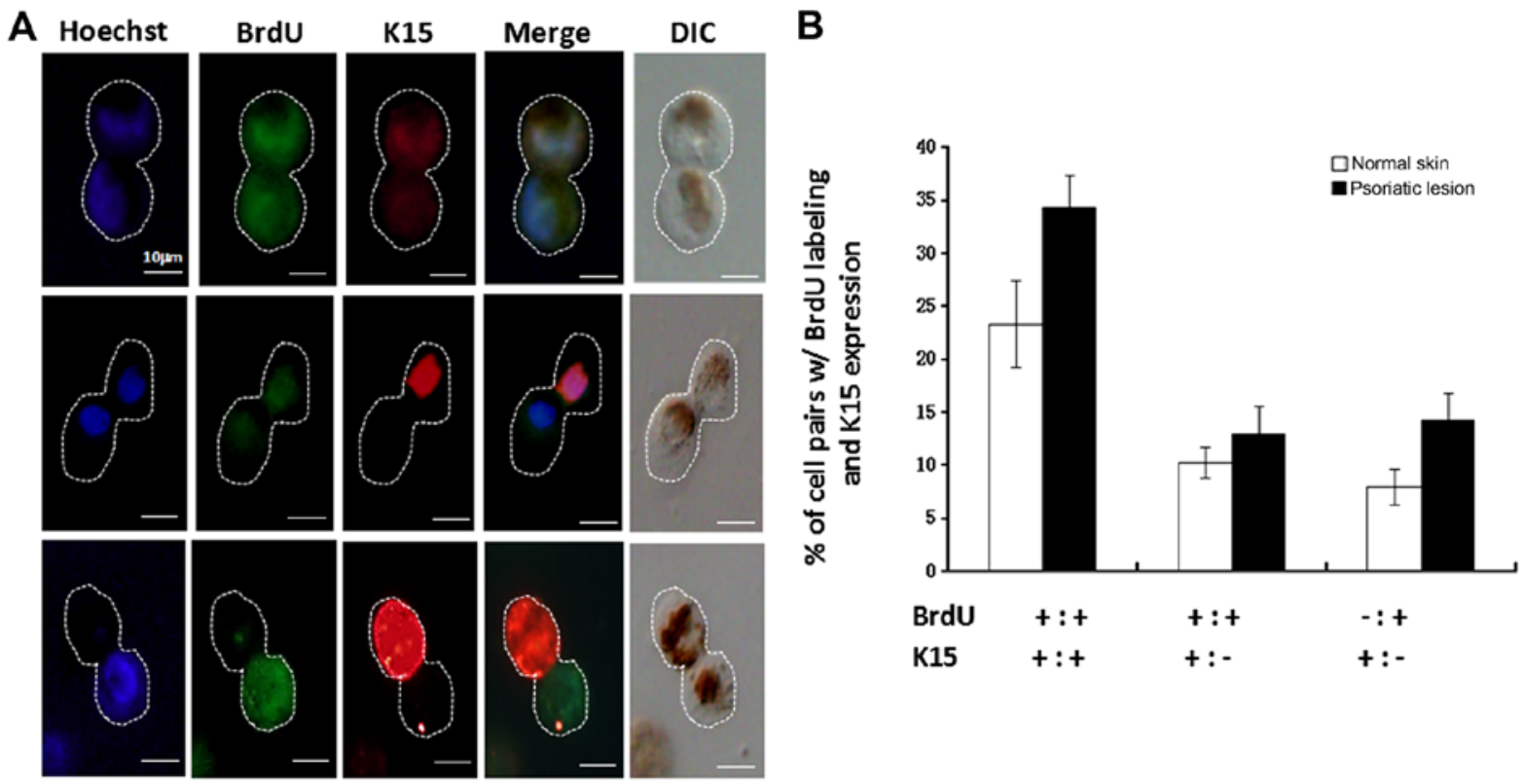

Figure 5. Determination of the transition from stem cells to transit-amplifying (TA) cells in BrdU pulse-chased anaphase cells using dual immunostaining for BrdU segregation and K15 expression. (A) Primary keratinocytes were cultured and pulse-chased with BrdU as described in Fig. 3. Cell pairs were co-immunostained for BrdU and K15. Depicted is a representative pair of cells demonstrating K15 expression and symmetric BrdU staining (top row), one daughter cell expressing K15 and symmetric BrdU staining (middle row), and one daughter cell expressing K15 and asymmetric BrdU staining (bottom row). The slides were counterstained with Hoechst 33258 to identify nuclei. Immunostaining was observed using an Olympus IX71 fluorescence microscope. Representative anaphase cells are shown in differential interference contrast (DIC) (furthest right panel), melanin pigment (brownish) was also visualized in the cells, which potentially affects the nuclear staining by Hoechst 33258 dye. Note that DNA template strands (BrdU-unlabeled) segregate to the less-differentiated progeny (K15-positive cells). The dotted lines delineate one cell pair each. Scale bar, $10 \mu \mathrm{m}$. (B) The percentage of cell pairs presenting asymmetric inheritance of template DNA strands and K15 expression. Data represent the means \pm SD from 3 independent experiments. " $\mathrm{P}<0.05$, compared to normal keratinocytes.

edge zone of the epithelial sheet in the absence of cytokine stimulation. However, following exposure to IL-17A and IL-22, both markers were expressed by cells throughout the epithelial tongue, and integrin $\beta 1$ was very brightly labeled in the inner cells close to the explant (Fig. 6C). Likewise, western blotting revealed that the expression of K15 and integrin $\beta 1$ was significantly upregulated in cells stimulated with both cytokines under low $(0.06 \mathrm{mM})$ and high $(1.3 \mathrm{mM})$ calcium conditions compared with untreated controls, and those 2 cytokines also exhibited a mild synergistic action (Fig. 7). These findings demonstrate that IL-17A and IL-22 play critical roles in maintaining an undifferentiated stem-cell like phenotype in basal keratinocytes.

\section{Discussion}

The emerging view is that psoriasis is a $\mathrm{T}$ cell-driven inflammatory skin disease characterized by keratinocyte hyperproliferation and aberrant terminal differentiation in sync with lymphocytic infiltrates $(1,10)$. In spite of persistent keratinocyte hyperproliferation, the constant maintenance of a single intact basal-cell layer has been noted as a distinct morphological characteristic of psoriatic epidermal hyperplasia $(4,24)$. Within the limited space of the basal layer, two types of proliferative keratinocytes, stem cells and TA cells, are aligned together and are attached to the underlying basement membrane. It has been proposed that the integrin $\beta 1$ expression level on the cell surface of basal keratinocytes is a key determinant which controls the transition of cells from the basal layer to the suprabasal layer $(25,26)$. Stem cells expressing higher levels of integrin $\beta 1$ than TA cells firmly adhere to the basement membrane, whereas TA cells have downregulated expression of integrin $\beta 1$ and thus lose their adherent ability. These integrin $\beta 1$-deficient TA cells then exit the basal layer and enter the suprabasal layer, and the terminal differentiation program is executed simultaneously, turning K15 transcription off and K10 transcription on (27). It has further been proposed that the proliferation taking place in the basal stem cells is a prerequisite for replenishing the enlarged compartment of TA cells in psoriatic epidermis $(28,29)$. The immunohistochemical data in the present study indicate that excessive expansion of the TA cell (K15-negative/integrin $\beta 1$-low/Ki67-positive) population indeed existed in the lesional psoriatic skin sections. BrdU-labeled mitotic basal cells in the mouse model of IMQ-induced psoriasis-like dermatitis were significantly increased compared with those treated with the control cream $(\mathrm{P}<0.05)$, and two types (perpendicular and parallel) of asymmetric cell division were also discerned in the inflamed skin of the mice (Fig. 3). In addition, the results of our in vitro analyses for template-DNA strand segregation in trypsin-dissociated epidermal cells using BrdU pulse-chase labeling are compatible with our assumption that stem cell division exists in the psoriatic epidermis (30). An increased percentage of asymmetric segregation of BrdU was noted in the cell pairs of psoriatic epidermal cells, whereas only a small proportion was noted in normal epidermal cells $(\mathrm{P}<0.001)$. The template DNA (BrdU-unlabeled strand) always segregated to the daughter cell, which retains K15 expression, indicating that psoriatic stem cell division also complies with the immortal strand hypothesis prediction that the cell inheriting the older template is the more undifferentiated cell, as reflected by the expression of K15. 


\section{A}

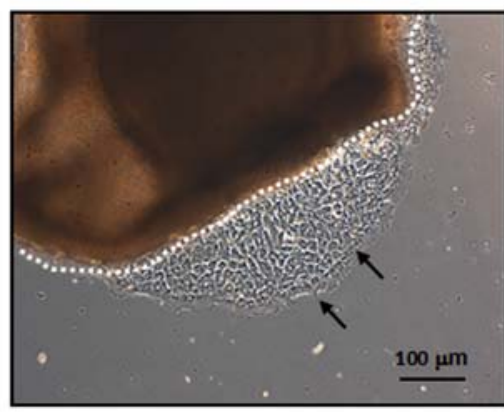

B
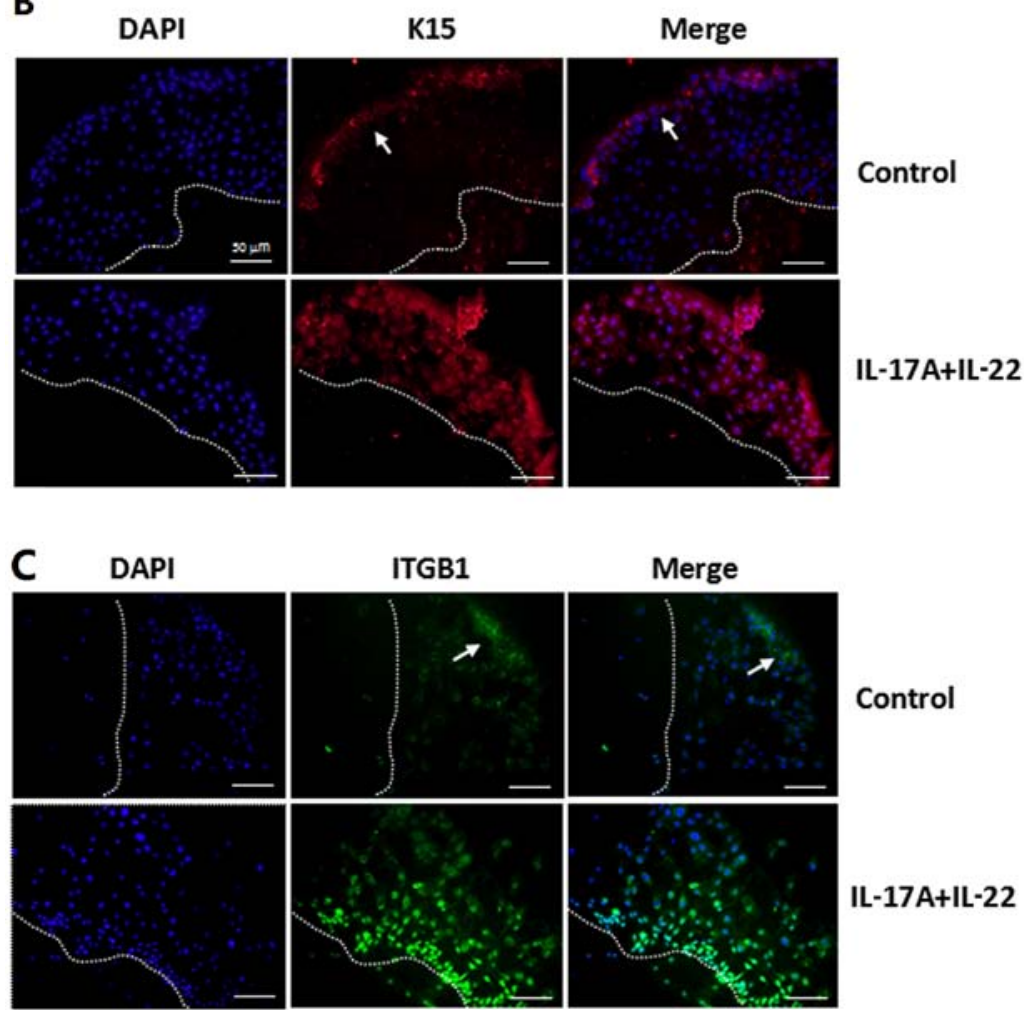

Control

IL-17A+IL-22

Figure 6. Effects of interleukin (IL)-17A and IL-22 on the expression of stem-cell markers in passage-one epidermal sheets. The epidermal sheet was prepared as described in Materials and methods. (A) After 3-5 days of cultivation, passage-one epidermal cells had grown from the edge of the skin explant to form a tongue-like epidermal sheet. The dotted line indicates the edge of the skin explant. Arrows indicate the leading-edge zone of the epidermal sheet. The epidermal sheet was treated with recombinant IL-17A $(10 \mathrm{ng} / \mathrm{ml})$ and IL-22 $(10 \mathrm{ng} / \mathrm{ml})$ for $24 \mathrm{~h}$. Shifts in the expression profiles of (B) K15 and (C) integrin $\beta 1$ (ITGB1) in the epidermal sheet were examined by immunofluorescence staining. The slides were counterstained with DAPI to identify nuclei. Immunostaining was observed using an Olympus IX71 fluorescence microscope. Scale bar, $50 \mu \mathrm{m}$.

The percentage of cells expressing K15 and asymmetrically labeled with $\mathrm{BrdU}\left(\mathrm{BrdU}^{-} / \mathrm{K}^{+} 5^{+}\right.$; $\left.\mathrm{BrdU}^{+} / \mathrm{K}^{-} 5^{-}\right)$is increased in psoriatic keratinocytes compared with normal cells $(\mathrm{P}<0.05)$. The percentage of cells expressing K15 that were symmetrically labeled with $\mathrm{BrdU}\left(\mathrm{BrdU}^{+} / \mathrm{K} 15^{+} ; \mathrm{BrdU}^{+} / \mathrm{K} 15^{+}\right)$was also increased in psoriatic keratinocytes compared with normal cells $(\mathrm{P}<0.01)$. These data reconfirm that both symmetric and asymmetric cell division contribute to the excessive expansion of the TA cell compartment in psoriatic epidermis (Fig. 5).

Previous studies have examined the role of Th17 cells in psoriatic epidermal hyperplasia $(9,10,18)$. Th17 cells have been reported to co-synthesize large amounts of IL-17A and IL-22, which disrupt keratinocyte terminal differentiation and enhance immune cell infiltration in psoriasis $(9,10)$. Several lines of evidence indicate that IL-22 (with or without IL-17) exerts an inhibitory effect on keratinocyte differentiation $(31,32)$. Our data demonstrate that upon stimulation with IL-17A and IL-22, the immunostaining pattern of $\mathrm{K} 15$ and integrin $\beta 1$ is changed in cultured epithelial cell sheets from the leading-edge zone to the entire sheet, as illustrated in Fig. 6. Similar results were obtained by western blotting, i.e., that undifferentiated markers (K15 and integrin $\beta 1$ ) are upregulated while differentiation markers (K10 and filaggrin) are downregulated in cultured keratinocytes stimulated by IL-17A and IL-22, respectively. As previously noted, even under high calcium $\left(1.2 \mathrm{mM} \mathrm{CaCl}_{2}\right)$ conditions, the differentiated marker pattern of keratinocytes is also suppressed by both cytokines (33). Surprisingly, the expression of the proliferative marker Ki67 was not upregulated in cells treated with IL-17A and IL-22 (Fig. 7), indicating that no proliferation-stimulating effect of IL-17A and IL-22 was achieved in cultured monolayers of keratinocytes, which is inconsistent with a previous report that IL-17A and IL-22 
A

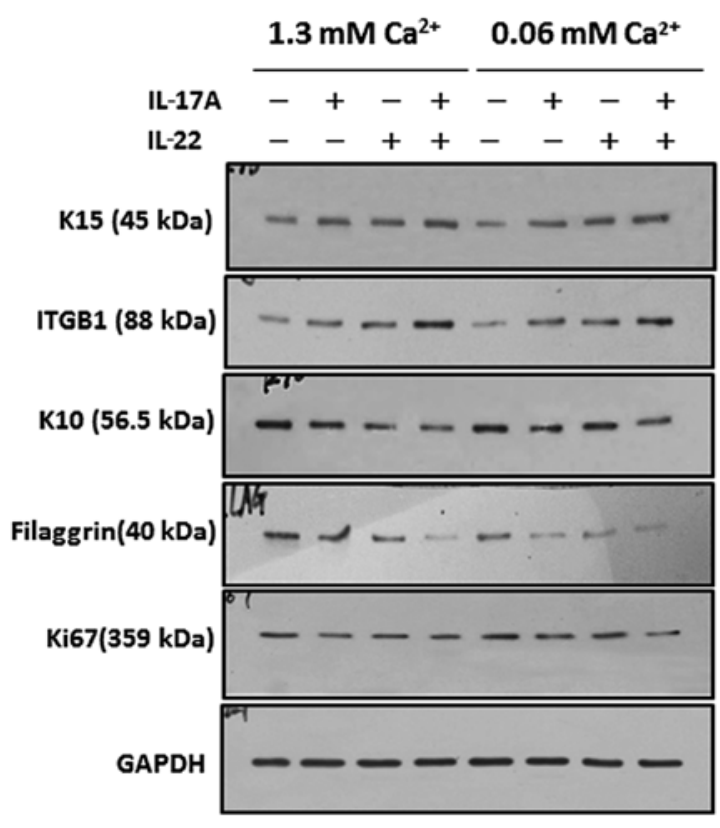

B
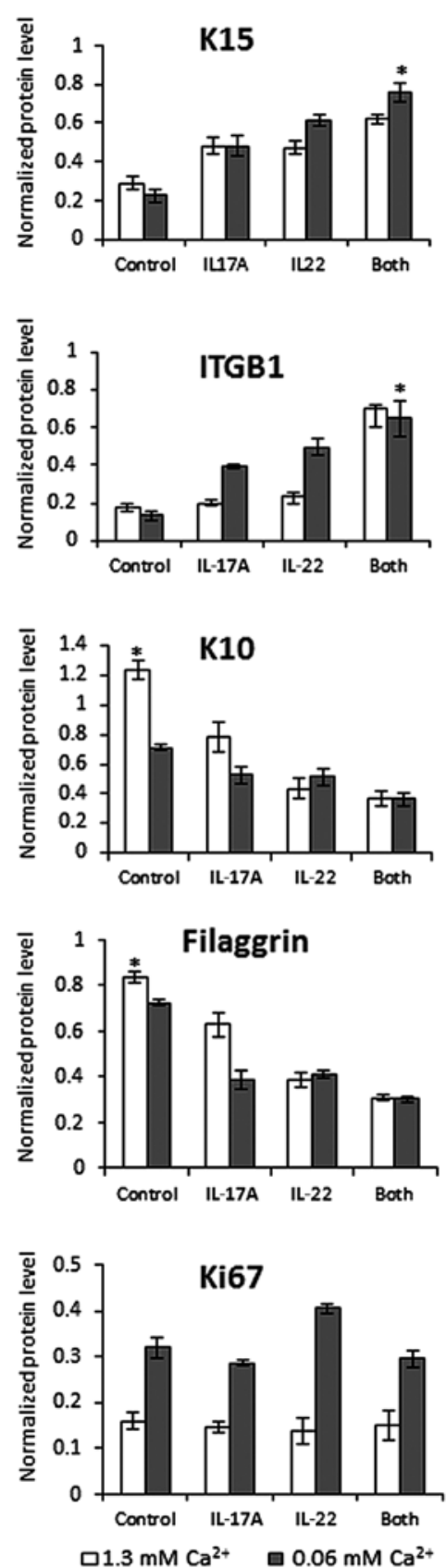

Figure 7. Effects of interleukin (IL)-17A and IL-22 on the expression of differentiation-related markers in cultured keratinocytes. Protein levels of undifferentiation (K15 and integrin $\beta 1$ ) and differentiation (K10 and filaggrin)-related markers were measured by western blotting. The cells were seeded into 6-well culture plates, and on the following day were treated with or without recombinant IL-17A (10 ng/ml) and IL-22 (10 ng/ml) at calcium concentrations of 0.06 and $1.3 \mathrm{mM}$ for $24 \mathrm{~h}$. After treatment, the cells were lysed using extraction buffer. Equal amounts ( $20 \mu \mathrm{g} / \mathrm{lane})$ of each protein extract were resolved using $10 \%$ SDS-PAGE. The protein loading variations were determined by western blotting with an anti-GAPDH antibody. (A) Representative blots are shown. (B) The protein level of each marker was quantified by densitometry and normalized to GAPDH, as shown in the histograms. Data are represented as the means \pm SD from 3 independent experiments. ${ }^{*} \mathrm{P}<0.05$, treatment groups compared with controls.

induced keratinocyte hyperproliferation in a three-dimensional skin equivalent model (10).

Taken together, these data provide unequivocal evidence that asymmetric basal stem-cell division contributes to psoriatic epidermal hyperplasia, which complies with the immortal strand hypothesis for the purpose of minimizing potential lethal mutations during the increased proliferation of psoriatic keratinocytes. We suggest that IL-17 and IL-22 cytokines play critical roles in regulating the expression of stem cell markers and in controlling the transition rate of cells from the basal layer to the suprabasal layer. Inhibition of hyperactive stem cells represents an intriguing potential therapeutic target to combat recalcitrant epidermal hyperplasia in psoriasis.

\section{Acknowledgements}

This study was supported by grants from the National Natural Science Foundation of China (nos. 8107138 and 81371717). The authors wish to thank Dr Vincent J. Hearing of the National Institutes of Health, Bethesda, MD, USA, for manuscript editing. 


\section{References}

1. Nestle FO, Kaplan DH and Barker J: Psoriasis. N Engl J Med 361 496-509, 2009.

2. Capon F, Burden AD, Trembath RC and Barker JN: Psoriasis and other complex trait dermatoses: from loci to functional pathways. J Invest Dermatol 132: 915-922, 2012.

3. Di Cesare A, Di Meglio P and Nestle FO: The IL-23/Th17 axis in the immunopathogenesis of psoriasis. J Invest Dermatol 129: $1339-1350,2009$

4. Leigh IM, Pulford KA, Ramaekers FC and Lane EB: Psoriasis: maintenance of an intact monolayer basal cell differentiation compartment in spite of hyperproliferation. Br J Dermatol 113 53-64, 1985.

5. Ma HL, Liang S, Li J, Napierata L, Brown T, Benoit S, Senices M, Gill D, Dunussi-Joannopoulos K, Collins M, et al: IL-22 is required for Th17 cell-mediated pathology in a mouse model of psoriasis-like skin inflammation. J Clin Invest 118: 597-607, 2008.

6. van der Fits L, Mourits S, Voerman JS, Kant M, Boon L, Laman JD, Cornelissen F, Mus AM, Florencia E, Prens EP and Lubberts E: Imiquimod-induced psoriasis-like skin inflammation in mice is mediated via the IL-23/IL-17 axis. J Immunol 182 : 5836-5845, 2009.

7. Nickoloff BJ: Keratinocytes regain momentum as instigators of cutaneous inflammation. Trends Mol Med 12: 102-106, 2006.

8. Eyerich S, Eyerich K, Cavani A and Schmidt-Weber C: IL-17 and IL-22: siblings, not twins. Trends Immunol 31: 354-361, 2010.

9. Nograles KE, Zaba LC, Guttman-Yassky E, Fuentes-Duculan J, Suárez-Fariñas M, Cardinale I, Khatcherian A, Gonzalez J, Pierson KC, White TR, et al: Th17 cytokines interleukin (IL)-17 and IL-22 modulate distinct inflammatory and keratinocyte-response pathways. Br J Dermatol 159: 1092-1102, 2008.

10. Guerrero-Aspizua S, García M, Murillas R, Retamosa L, Illera N, Duarte B, Holguín A, Puig S, Hernández MI, Meana A, et al: Development of a bioengineered skin-humanized mouse model for psoriasis: dissecting epidermal-lymphocyte interacting pathways. Am J Pathol 177: 3112-3124, 2010

11. Beck B and Blanpain C: Mechanisms regulating epidermal stem cells. EMBO J 31: 2067-2075, 2012.

12. Blanpain $\mathrm{C}$ and Fuchs E: Epidermal homeostasis: a balancing act of stem cells in the skin. Nat Rev Mol Cell Biol 10: 207-217, 2009.

13. Dazard JE, Piette J, Basset-Seguin N, Blanchard JM and Gandarillas A: Switch from p53 to MDM2 as differentiating human keratinocytes lose their proliferative potential and increase in cellular size. Oncogene 19: 3693-3705, 2000.

14. Arwert EN, Lal R, Quist S, Rosewell I, van Rooijen N and Watt FM: Tumor formation initiated by nondividing epidermal cells via an inflammatory infiltrate. Proc Natl Acad Sci USA 107: 19903-19908, 2010

15. Janich P, Pascual G, Merlos-Suárez A, Batlle E, Ripperger J, Albrecht U, Cheng HY, Obrietan K, Di Croce L and Benitah SA: The circadian molecular clock creates epidermal stem cell heterogeneity. Nature 480: 209-214, 2011.

16. Guo A and Jahoda CA: An improved method of human keratinocyte culture from skin explants: cell expansion is linked to markers of activated progenitor cells. Exp Dermatol 18: 720-726, 2009.

17. Gutowska-Owsiak D, Schaupp AL, Salimi M, Selvakumar TA, McPherson T, Taylor S and Ogg GS: IL-17 downregulates filaggrin and affects keratinocyte expression of genes associated with cellular adhesion. Exp Dermatol 21: 104-110, 2012.
18. Guilloteau K, Paris I, Pedretti N, Boniface K, Juchaux F, Huguier V, Guillet G, Bernard FX, Lecron JC and Morel F: Skin inflammation induced by the synergistic action of IL-17A, IL-22, oncostatin M, IL- $1 \alpha$, and TNF- $\alpha$ recapitulates some features of psoriasis. J Immunol 184: 5263-5270, 2010.

19. Shinin V, Gayraud-Morel B, Gomès D and Tajbakhsh S: Asymmetric division and cosegregation of template DNA strands in adult muscle satellite cells. Nat Cell Biol 8: 677-687, 2006.

20. Conboy MJ, Karasov AO and Rando TA: High incidence of non-random template strand segregation and asymmetric fate determination in dividing stem cells and their progeny. PLoS Biol 5: e102, 2007.

21. Luo LF, Shi Y, Zhou Q, Xu SZ and Lei TC: Insufficient expression of the melanocortin-1 receptor by human dermal fibroblasts contributes to excess collagen synthesis in keloid scars. Exp Dermatol 22: 764-766, 2013.

22. Waseem A, Dogan B, Tidman N, Alam Y, Purkis P, Jackson S, Lalli A, Machesney M and Leigh IM: Keratin 15 expression in stratified epithelia: downregulation in activated keratinocytes. J Invest Dermatol 112: 362-369, 1999.

23. Troy TC, Arabzadeh A and Turksen K: Re-assessing K15 as an epidermal stem cell marker. Stem Cell Rev 7: 927-934, 2011.

24. Körver JE, Vissers WH, van Rens DW, Pasch MC, van Erp PE, Boezeman JB and van De Kerkhof PC: A double-blind, randomized quantitative comparison of calcitriol ointment and calcipotriol ointment on epidermal cell populations, proliferation and differentiation. Br J Dermatol 156: 130-137, 2007.

25. Watt FM: Epidermal stem cells: markers, patterning and the control of stem cell fate. Philos Trans R Soc Lond B Biol Sci 353: 831-837, 1998.

26. Grabe $\mathrm{N}$ and Neuber K: Simulating psoriasis by altering transit amplifying cells. Bioinformatics 23: 1309-1312, 2007.

27. Jones P and Simons BD: Epidermal homeostasis: do committed progenitors work while stem cells sleep? Nat Rev Mol Cell Biol 9: 82-88, 2008.

28. Truzzi F, Marconi A, Atzei P, Panza MC, Lotti R, Dallaglio K, Tiberio R, Palazzo E, Vaschieri C and Pincelli C: p75 neurotrophin receptor mediates apoptosis in transit-amplifying cells and its overexpression restores cell death in psoriatic keratinocytes. Cell Death Differ 18: 948-958, 2011

29. Kawashima K, Doi H, Ito Y, Shibata MA, Yoshinaka R and Otsuki Y: Evaluation of cell death and proliferation in psoriatic epidermis. J Dermatol Sci 35: 207-214, 2004.

30. Gündüz K, Demireli P, Vatansever S and Inanir I: Examination of bcl-2 and p53 expressions and apoptotic index by TUNEL method in psoriasis. J Cutan Pathol 33: 788-792, 2006.

31. Boniface K, Bernard FX, Garcia M, Gurney AL, Lecron JC and Morel F: IL-22 inhibits epidermal differentiation and induces proinflammatory gene expression and migration of human keratinocytes. J Immunol 174: 3695-3702, 2005.

32. Rabeony H, Petit-Paris I, Garnier J, Barrault C, Pedretti N, Guilloteau K, Jegou JF, Guillet G, Huguier V, Lecron JC, et al: Inhibition of keratinocyte differentiation by the synergistic effect of IL-17A, IL-22, IL-1 $\alpha$, TNF $\alpha$ and oncostatin M. PLoS One 9: e101937, 2014.

33. Xie Z, Singleton PA, Bourguignon LY and Bikle DD: Calcium-induced human keratinocyte differentiation requires src- and fyn-mediated phosphatidylinositol 3-kinase-dependent activation of phospholipase C-gamma1. Mol Biol Cell 16: 32363246,2005 